\title{
Joint Waveform and Guidance Control Optimization by Statistical Linearisation for Target Rendezvous
}

\author{
Alessio Benavoli ${ }^{1}$ \\ Department of Statistics, \\ School of Computer Science and Statistics \\ Trinity College Dublin, Ireland \\ benavoli@gmail.com
}

\author{
Alessio Balleri ${ }^{2}$ \\ Centre for Electronic Warfare, Information \\ and Cyber, Cranfield University \\ Defence Academy of the UK \\ Shrivenham, SN6 8LA \\ a.balleri@cranfield.ac.uk
}

\author{
Alfonso Farina ${ }^{3,2}$ \\ Selex-ES (retired) \\ Rome, Italy \\ alfonso.farina@outlook.it
}

\begin{abstract}
The algorithm proposed in this paper jointly selects the transmitted waveform and the control input so that a radar sensor on a moving platform can prosecute a target by minimising a predefined cost that accounts for the energy of the transmitted radar signal, the energy of a platform control input and the relative position error between the platform and the target. The cost is a function of the waveform design and control input. The algorithm extends the existing Joint Waveform Guidance and Control Optimization (JWGCO) solution to nonlinear equations to account for the dependency of the radar measurement accuracies on Signal to Noise Ratio (SNR) and, as a consequence, on the target position. The performance of the proposed solution based on statistical linearisation is assessed with a set of simulations for a pulsed Doppler radar transmitting linearly frequency modulated chirps.

Index Terms-Cognitive rendezvous, adaptive waveform design, cognitive radar, Fisher Information Matrix (FIM), Cramér-Rao Lower Bound (CRLB), Linear Quadratic Gaussian control (LQG), Kalman Filter (KF), Joint Waveform and Guidance Control Optimization (JWGCO).
\end{abstract}

\section{INTRODUCTION}

The improvement of technology and the pressing need for sensors to become increasingly more flexible, adaptive, and ultimately include some degree of cognition, is resulting in radar systems that are becoming more and more capable to adapt their parameters from scan to scan to optimise the sensor performance.

Cognitive radar systems are radar that continuously learn about the environment through active interactions with the environment itself and, as a result, continually update the receiver and the transmitting parameters, in an intelligent manner, with relevant information (the perception action cycle). The whole radar system is a dynamic closed feedback loop between the transmitter, environment and receiver. Cognition can be achieved with the use of prior knowledge on the environment, which represents the long-term memory of the receiver, and the short-term memory developed by the receiver on the fly.

In recent years, there has been growing research interest to develop solutions that can increase the level of cognition of radar systems [1], [2]. The largest portion of research published on cognitive radar presents solutions to adapt the transmitter parameters in response to the surrounding environment under the tracking framework (e.g. [3]-[10]).

Despite previous work on tracking by cognitive radar, very little research has been focused on how radar cognition can be applied to guidance control for target rendezvous. There has been prior work on path planning, investigating optimal trajectories of receivers in a multistatic network for tracking, with no attempt to achieve guidance control and apply constraints to each receiving platform trajectory to satisfy a predefined cost function [11]. The key challenge of studying cognition applied to rendezvous problems is the requirement of optimal solutions that handle estimation theory and platform guidance control jointly to ensure the trajectory of a platform ends at the target.

Optimal Waveform Diversity (WD) and platform control for target rendezvous are techniques which have been mostly researched separately in the past. One of the first papers on optimal guidance for rendezvous investigated the case of a sensor on the ground that delivered optimal guidance to an interceptor on the basis of the estimated trajectories of the interceptor and the target to reach [12]. An algorithm to automatically select the transmitted waveform design from scan to scan to guide an airborne platform towards a target was presented in [13]. In this paper, the fore-active control technique proposed in [3] was used to select the transmitted waveform, so to minimise the innovation matrix (or residual) of the Kalman Filter (KF), and a standard Linear Quadratic Gaussian control (LQG) technique was employed to guide the platform towards the target. However, in this work, the foreactive technique and LQG control were applied independently with no attempt to demonstrate the existence and find the joint optimal solution. An optimal solution, named the JWGCO, was presented in [14] to achieve joint optimal Waveform Diversity (WD) and guidance control for rendezvous. The JWGCO algorithm minimises a cost function that depends on both the parameters of the transmitted radar waveform and the input guidance control so that the waveform and guidance control input selection, for a linear system, can be jointly and simultaneously optimised. It was demonstrated that for a linear Gaussian system (in the dynamic and measurement equations), when the measurement noise is independent of 
the state, the joint optimisation problem can be simplified into two independent optimisations, one that depends only on the waveform parameters and the other that depends solely on the control input. The solution was provided in a closedform generalising the linear KF theory and the LQG control approach.

In this paper, a solution based on Statistical Linearisation (SL) is presented that extends the JWGCO solution to the non-linear measurement case. The JWGCO/SL solution accounts for the dependency of the radar measurement accuracies on the target position and, as a direct consequence, on the Signal to Noise Ratio.

\section{INTERCEPTOR-TARGET DYNAMICAL SYSTEM}

The relative polar coordinates between the interceptor and

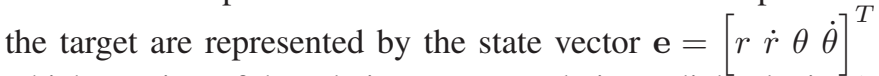
which consists of the relative range $r$, relative radial velocity $\dot{r}$, relative angle $\theta$ and relative angle rate $\dot{\theta}$ between the platform and the target. The state equations are [15], [16]:

$$
\mathbf{e}_{k}=\mathbf{F}_{k-1} \mathbf{e}_{k-1}+\mathbf{B}_{k-1} \mathbf{u}_{k-1}+\mathbf{w}_{k-1},
$$

where $\mathbf{e}_{k}$ is the state vector, $\mathbf{F}_{k-1}$ is the matrix dynamics and $\mathbf{w}_{k-1}$ is Gaussian with zero-mean and covariance matrix $\mathbf{Q}_{k-1}$. The term $\mathbf{B}_{k-1} \mathbf{u}_{k-1}$ is the control action at each step: the input vector control $\mathbf{u}_{k-1}$ is combined linearly with a matrix $\mathbf{B}_{k-1}$ before being applied to the dynamics equations. We consider the case with

$$
\mathbf{F}=\left(\begin{array}{cccc}
1 & T & 0 & 0 \\
0 & 1 & 0 & 0 \\
0 & 0 & 1 & T \\
0 & 0 & 0 & 1
\end{array}\right), \quad \mathbf{Q}=\left(\begin{array}{ll}
\mathbf{Q}_{r} & \mathbf{0} \\
\mathbf{0} & \mathbf{Q}_{\theta}
\end{array}\right)
$$

and

$\mathbf{Q}_{r}=\sigma_{1}^{2}\left(\begin{array}{ll}T^{3} / 3 & T^{2} / 2 \\ T^{2} / 2 & T\end{array}\right), \quad \mathbf{Q}_{\theta}=\sigma_{2}^{2}\left(\begin{array}{ll}T^{3} / 3 & T^{2} / 2 \\ T^{2} / 2 & T\end{array}\right)$,

$\sigma_{1}^{2}=0.05, \sigma_{2}^{2}=0.08$ and $T=1$ s and $\mathbf{B}=\mathbf{I}_{4 \times 4}$. At each time $k$ the interceptor transmits a waveform, defined by a vector of parameters $\gamma_{k}$ (e.g., bandwidth, duration etc.), to obtain a measurement $\mathbf{y}_{k}$ of its relative state $\mathbf{e}_{k}$ with respect to the target. We consider the following measurement equation:

$$
\mathbf{y}_{k}=\mathbf{H e}_{k}+\mathbf{v}_{k}\left(\mathbf{e}_{k}, \gamma_{k}\right)=\left[\begin{array}{c}
r_{k} \\
\dot{r}_{k} \\
\theta_{k}
\end{array}\right]+\mathbf{v}_{k}\left(\mathbf{e}_{k}, \gamma_{k}\right),
$$

where $\mathbf{v}_{k}\left(\mathbf{e}_{k}, \gamma_{k}\right)$ is a Gaussian noise with zero-mean and a covariance matrix $\mathbf{N}_{k}\left(\mathbf{e}_{k}, \gamma_{k}\right)$. Note that the covariance matrix $\mathbf{N}_{k}\left(\mathbf{e}_{k}, \gamma_{k}\right)$ depends on the accuracy of the transmitted waveform $s\left(t, \gamma_{k}\right)$, whose design is fully described by the vector of parameters $\gamma_{k}$ (defining the waveform features, such as duration, bandwidth and time-frequency curvature) and on the accuracy of the measurement of angle. It also depends on the state $\mathbf{e}_{k}$ due to the SNR as detailed below. We write

$$
\mathbf{N}_{k}\left(\mathbf{e}_{k}, \gamma_{k}\right)=\left(\begin{array}{cc}
\hat{\mathbf{N}}_{k}\left(\mathbf{e}_{k}, \boldsymbol{\gamma}_{k}\right) & \mathbf{0}_{2 \times 1} \\
\mathbf{0}_{1 \times 2} & \sigma_{t}^{2}
\end{array}\right)
$$

where $\hat{\mathbf{N}}_{k}\left(\mathbf{e}_{k}, \gamma_{k}\right)$ corresponds to the Cramér-Rao Lower Bound (CRLB), relative to the task of joint estimation of range and radial velocity between a sensor and a target, when the sensor transmits the signal $s\left(t, \gamma_{k}\right)$. The CRLB is obtained as the inverse of the Fisher Information Matrix (FIM).

For a train of $N_{p}$ Gaussian Linear Frequency Modulated (LFM) chirps with duration $T=2 \lambda_{G}$ and bandwidth $B=2 b_{G} \lambda_{G} / \pi\left(\gamma_{k}=\left[\lambda_{G} \cdot b_{G}\right]\right)$, the FIM of time delay and Doppler can be approximated as [17]

$\mathbf{J}=\operatorname{SNR}\left(r, \lambda_{G}\right)\left(\begin{array}{lr}\frac{1}{2 \lambda_{G}^{2}}+2 \lambda_{G}^{2} b_{G}^{2} & 2 \pi \lambda_{G}^{2} b_{G} \\ 2 \pi \lambda_{G}^{2} b_{G} & 2 \pi^{2} \lambda_{G}^{2}+\frac{1}{3} \pi^{2} \mathrm{PRI}^{2}\left(N_{p}^{2}-1\right)\end{array}\right)$

and

$$
\hat{\mathbf{N}}_{k}\left(\mathbf{e}_{k}, \gamma_{k}\right)=\left(\begin{array}{cc}
\frac{c^{2}}{4} & \frac{c \lambda}{4} \\
\frac{c \lambda}{4} & \frac{\lambda^{2}}{4}
\end{array}\right) \circ \mathbf{J}^{-1}
$$

where $c=3 \cdot 10^{8} \mathrm{~m} / \mathrm{s}$ is the speed of light, $\lambda$ is the wavelength at the carrier frequency $f_{0}$ and $\circ$ denotes the component-wise product. The Signal to Noise Ratio (SNR) is a function of the state $r$ and the waveform parameter $\lambda_{G}$ and, neglecting losses, can be expressed as

$$
\operatorname{SNR}\left(r, \lambda_{G}\right)=2 N_{P} \frac{P_{p k} \sqrt{\pi \lambda_{G}^{2}} G^{2} \sigma \lambda^{2}}{(4 \pi)^{3} r^{4} k T_{e}},
$$

where $P_{p k}$ is the transmitted peak power, $G$ is the antenna gain, $\sigma$ is the target Radar Cross Section (RCS), $k$ is the Boltzmann's constant and $T_{e}$ is the equivalent noise temperature of the receiver ${ }^{1}$. Finally, it is observed that the transmitted energy for the train of $N_{p}$ Gaussian chirps can be expressed as

$$
\psi_{s}=N_{P} P_{p k} \sqrt{\pi \lambda_{G}^{2}}
$$

and is also a function of the waveform parameter $\lambda_{G}$.

\section{WAVEFORM AND GUIDANCE CONTROL PROBLEM}

The goal of the interceptor is to minimise a quadratic cost:

$$
J\left(\mathbf{e}_{o}, \mathbf{u}_{0: N-1}, \gamma_{1: N}\right)=\mathcal{E}\left(\sum_{k=0}^{N} \ell\left(k, \mathbf{e}_{k}, \mathbf{u}_{k}, \gamma_{k}\right)\right),
$$

with respect to $\mathbf{u}_{0: N-1}=\left\{\mathbf{u}_{o}, \ldots, \mathbf{u}_{N-1}\right\}$ and $\gamma_{1: N}=$ $\left\{\gamma_{1}, \ldots, \gamma_{N}\right\}$, over the temporal horizon $N$, with

$$
\ell\left(k, \mathbf{e}_{k}, \mathbf{u}_{k}, \gamma_{\boldsymbol{k}}\right)=\mathbf{e}_{k}^{T} \mathbf{E}_{k} \mathbf{e}_{k}+\mathbf{u}_{k}^{T} \mathbf{R}_{k} \mathbf{u}_{k}+\alpha_{k} \psi_{s}\left(\gamma_{\boldsymbol{k}}\right),
$$

for $k<N$ and

$\ell\left(N, \mathbf{e}_{N}, \mathbf{u}_{N}, \gamma_{N}\right)=\mathbf{e}_{N}^{T} \mathbf{E}_{N} \mathbf{e}_{N}+\operatorname{Tr}\left(\mathbf{E}_{N} \mathbf{P}_{N \mid N}\right)+\alpha_{N} \psi_{s}\left(\gamma_{N}\right)$,

for $k=N$, where $\mathcal{E}(\ldots)$ denotes the expectation w.r.t. the initial state and the sequence of measurement and process noises. Note that $\mathbf{E}_{k}, \mathbf{R}_{k}$ are Positive Semi-Definite (PSD) matrices that define the weights of the cost function: the matrices $\mathbf{R}_{k}$ weigh the control effort, while $\mathbf{E}_{k}$ weight the

\footnotetext{
${ }^{1}$ The equivalent noise temperature of the receiver can also be expressed as $T_{e}=T_{0} F_{e}$, where $T_{0}=290 \mathrm{~K}^{\circ}$ and $F_{e}$ is the receiver noise figure.
} 
control effectiveness; $\alpha_{k} \geq 0$ are additional scalar weights of the cost function that penalise a sensing cost term $\psi_{s}\left(\gamma_{k}\right) \geq 0$; and $\operatorname{Tr}\left(\mathbf{E}_{N} \mathbf{P}_{N \mid N}\right)$ is an additional terminal cost and $\mathbf{P}_{N \mid N}$ is the covariance of the terminal state $\mathbf{e}_{N}$. The goal is thus to keep $\mathbf{e}_{k}$ close to $\mathbf{0}$, particularly, at the final time $N$, using little control effort $\mathbf{u}_{k}$ and little sensing cost $\psi_{s}\left(\gamma_{k}\right)$.

Note that, the cost functions depends on $\gamma_{1: N}$ due to: (i) the sensing cost term $\psi_{s}\left(\gamma_{k}\right)$; (ii) the measurement noise $\left(\mathbf{v}_{k}\left(\gamma_{k}\right)\right)$ that contributes to the covariance matrix of the joint multivariate Gaussian distribution w.r.t. which the expectation in (10) is taken.

For fixed sensor parameters (fixed $\gamma_{1: N}$ ) and $\alpha_{k}=0$, the cost function in (10) coincides with that of the Linear Quadratic Gaussian (LQG) regulator problem (see for instance [18]). However, the solution of the above control problem is not the same as in LQG because of the nonlinearity in the measurement equation. The measurement $\mathbf{y}_{k}$ depends nonlinearly on the state $\mathbf{e}_{k}$ due to the dependence of the covariance of the measurement noise, $\mathbf{N}_{k}\left(\mathbf{e}_{k}, \gamma_{k}\right)$, on the range via the SNR, see (6)-(7). In this case, no closed form solution can be computed for the LQG control. In this paper, we propose an approximation based on the statistical linearisation approach.

\section{STATISTICAL LinEARISATION}

Whenever the kinematic and measurement model are nonlinear/non-Gaussian, the posterior distribution of the state cannot be computed analytically so approximations must be used. In this paper, we approximate the nonlinear measurement model using statistical linearisation [19]. In other words, we approximate the measurement model

$$
\mathbf{H e}_{k}+\mathbf{v}_{k}\left(\mathbf{e}_{k}, \gamma_{k}\right) \approx \mathbf{H e}_{k}+\boldsymbol{\xi}_{k}\left(\gamma_{k}\right),
$$

where $\boldsymbol{\xi}_{k}\left(\gamma_{k}\right) \sim \mathcal{N}\left(0, \Phi_{k}\right)$ (that is, Gaussian distributed with zero mean and variance $\boldsymbol{\Phi}_{k}$ ) does not depend on $\mathbf{e}_{k}$ and we determine the matrix $\boldsymbol{\Phi}_{k}$, which is a function of $\gamma_{k}$, by minimizing the Kullback-Leibler (KL) divergence between the true posterior and the approximating Gaussian model. Though the above linearisation is different from that used in [19]; in our case the covariance matrix $\mathbf{N}_{k}\left(\mathbf{e}_{k}, \gamma_{k}\right)$ depends on the state $\mathbf{e}_{k}$ while the measurement model is linear. This is the reason why we rederive the approximation in Theorem 1 which follows.

Let us focus on a fixed $k$ step for a point $\mathbf{e}_{k}$. We have that $^{2} p\left(\mathbf{y}_{k} \mid \mathbf{e}_{k}\right)=\mathcal{N}\left(\mathbf{y}_{k} ; \mathbf{H} \mathbf{e}_{k}, \mathbf{N}\left(\mathbf{e}_{k}, \boldsymbol{\gamma}_{k}\right)\right)$ and $p\left(\mathbf{e}_{k}\right)=$ $\mathcal{N}\left(\mathbf{e}_{k} ; \mathbf{e}_{o}, \mathbf{P}_{o}\right)$, where $\mathbf{e}_{o}=\hat{\mathbf{e}}_{k \mid k-1}$ and $\mathbf{P}_{o}=\mathbf{P}_{k \mid k-1}$. Observe that the joint distribution $p\left(\mathbf{y}_{k}, \mathbf{e}_{k}\right)=p\left(\mathbf{y}_{k} \mid \mathbf{e}_{k}\right) p\left(\mathbf{e}_{k}\right)$ is not Gaussian. We approximate the covariance of the noise so that $\boldsymbol{\xi}_{k}$ is a zero-mean Gaussian with covariance $\boldsymbol{\Phi}_{k}$ (that is a "linearization noise" whose variance depends on $k$ ) so that

$$
p\left(\mathbf{y}_{k}, \mathbf{e}_{k}\right) \approx q\left(\mathbf{y}_{k}, \mathbf{e}_{k}\right)=\mathcal{N}\left(\mathbf{y} ; \mathbf{H e}_{k}, \boldsymbol{\Phi}_{k}\right) p\left(\mathbf{e}_{k}\right) .
$$

\footnotetext{
${ }^{2}$ We use the notation $\mathcal{N}(x ; m, \Sigma)$ to denote the Gaussian PDF of the random variable $x$ with mean $m$ and covariance $\Sigma$.
}

Theorem 1. The optimal parameter $\boldsymbol{\Phi}_{k}$ that minimizes the $K L$ distance between the joint $p\left(\mathbf{y}_{k}, \mathbf{e}_{k}\right)$ and $q\left(\mathbf{y}_{k}, \mathbf{e}_{k}\right)$ :

$$
\min _{\mathbf{\Phi}_{k}} \int p\left(\mathbf{y}_{k}, \mathbf{e}_{k}\right) \ln \frac{p\left(\mathbf{y}_{k}, \mathbf{e}_{k}\right)}{q\left(\mathbf{y}_{k}, \mathbf{e}_{k}\right)} d \mathbf{e}_{k} d \mathbf{y}_{k}
$$

is given by

$$
\tilde{\boldsymbol{\Phi}}_{k}=\mathcal{E}\left(\mathbf{N}\left(\mathbf{e}_{k}, \gamma_{k}\right)\right),
$$

where $\mathcal{E}(\cdot)=\int(\cdot) \mathcal{N}\left(\mathbf{e}_{k} ; \mathbf{e}_{o}, \mathbf{P}_{o}\right) d \mathbf{e}_{k}$.

Proof. In what follows we drop the dependency on $k$ and on $\gamma_{k}$ to simplify the notation.

$$
\begin{aligned}
& \iint p(\mathbf{e}, \mathbf{y}) \ln \frac{p(\mathbf{e}, \mathbf{y})}{q(\mathbf{e}, \mathbf{y})} d \mathbf{e} d \mathbf{y} \\
& =\iint \mathcal{N}(\mathbf{y} ; \mathbf{H e}, \mathbf{N}(\mathbf{e})) p(\mathbf{e}) \ln \frac{\mathcal{N}(\mathbf{y} ; \mathbf{H e}, \mathbf{N}(\mathbf{e}))}{\mathcal{N}(\mathbf{y} ; \mathbf{H e}, \mathbf{\Phi})} d \mathbf{e} d \mathbf{y} \\
& =\iint \mathcal{N}(\mathbf{y} ; \mathbf{H e}, \mathbf{N}(\mathbf{e})) p(\mathbf{e}) \ln \mathcal{N}(\mathbf{y} ; \mathbf{H e}, \mathbf{N}(\mathbf{e})) d \mathbf{e} d \mathbf{y} \\
& -\iint \mathcal{N}(\mathbf{y} ; \mathbf{H e}, \mathbf{N}(\mathbf{e})) p(\mathbf{e}) \ln \mathcal{N}(\mathbf{y} ; \mathbf{H e}, \mathbf{\Phi}) d \mathbf{e} d \mathbf{y}
\end{aligned}
$$

The first term does not depend on $\boldsymbol{\Phi}$. We can then compute the derivative of the second term w.r.t. $\Phi$ which is

$$
\begin{aligned}
& \frac{\partial}{\partial \boldsymbol{\Phi}}\left(-\iint \mathcal{N}(\mathbf{y} ; \mathbf{H e}, \mathbf{N}(\mathbf{e})) p(\mathbf{e}) \ln \mathcal{N}(\mathbf{y} ; \mathbf{H e}, \boldsymbol{\Phi}) d \mathbf{e} d \mathbf{y}\right) \\
& =\frac{1}{2} \iint \mathcal{N}(\mathbf{y} ; \mathbf{H e}, \mathbf{N}(\mathbf{e})) p(\mathbf{e}) \frac{\partial}{\partial \boldsymbol{\Phi}} \ln (\operatorname{det}(2 \pi \boldsymbol{\Phi})) d \mathbf{e} d \mathbf{y} \\
& +\frac{1}{2} \iint \mathcal{N}(\mathbf{y} ; \mathbf{H e}, \mathbf{N}(\mathbf{e})) p(\mathbf{e}) \frac{\partial}{\partial \boldsymbol{\Phi}} \operatorname{Tr}\left(\mathbf{\Phi}^{-1} \omega \omega^{T}\right) d \mathbf{e} d \mathbf{y}
\end{aligned}
$$

with $\omega=\mathbf{y}-\mathbf{H e}$. The first integral is equal to $\frac{1}{2} \operatorname{Tr}\left(\mathbf{\Phi}^{-1}\right)$. The second integral:

$$
\begin{aligned}
& \iint \mathcal{N}(\mathbf{y} ; \mathbf{H e}, \mathbf{N}(\mathbf{e})) p(\mathbf{e}) \frac{\partial}{\partial \boldsymbol{\Phi}} \operatorname{Tr}\left(\mathbf{\Phi}^{-1} \omega \omega^{T}\right) d \mathbf{e} d \mathbf{y} \\
& =-\iint \mathcal{N}(\mathbf{y} ; \mathbf{H e}, \mathbf{N}(\mathbf{e})) p(\mathbf{e}) \operatorname{Tr}\left(\mathbf{\Phi}^{-2} \omega \omega^{T}\right) d \mathbf{e} d \mathbf{y}
\end{aligned}
$$

We replace $\omega=\mathbf{y}-\mathbf{H e}$ and so the integral becomes

$$
\begin{aligned}
& -\iint \mathcal{N}(\mathbf{y} ; \mathbf{H e}, \mathbf{N}(\mathbf{e})) p(\mathbf{e}) \operatorname{Tr}\left(\mathbf{\Phi}^{-2} \omega \omega^{T}\right) d \mathbf{e} d \mathbf{y} \\
& =-\int p(\mathbf{e}) \operatorname{Tr}\left(\boldsymbol{\Phi}^{-2} \mathbf{N}(\mathbf{e})\right) d \mathbf{e} \\
& =-\operatorname{Tr}\left(\boldsymbol{\Phi}^{-2} \int p(\mathbf{e}) \mathbf{N}(\mathbf{e}) d \mathbf{e}\right) .
\end{aligned}
$$

By imposing $\frac{\partial}{\partial \Phi}=0$ :

$$
\tilde{\boldsymbol{\Phi}}=\mathcal{E}(\mathbf{N}(\mathbf{e}))
$$

that ends the proof.

To compute the optimal parameter we need to solve the integral in (12). We can use any sigma-points method. In this paper we employ the Unscented Transform (UT) [20] with 7 sigma points and parameters $\beta=2, \alpha=0.001, \kappa=1{ }^{3}$

\footnotetext{
${ }^{3}$ These are standard parameters for UT.
} 
Therefore, we generate 7 sigma points $\chi_{1}, \ldots, \chi_{7}$ and weights $\omega_{1}, \ldots, \omega_{7}$ such that they match the moments $\mathbf{e}_{o}$ and $\mathbf{P}_{o}$, and employ them to compute the approximation:

$$
\tilde{\mathbf{\Phi}}_{k}=\mathcal{E}\left(\mathbf{N}\left(\mathbf{e}_{k}\right)\right) \approx \sum_{i=1}^{7} \omega_{i} \mathbf{N}\left(\chi_{i}\right)
$$

We use the approximating covariance matrix $\tilde{\boldsymbol{\Phi}}_{k}$, which is a function of the waveform parameters $\gamma_{k}$, to compute the optimal waveform parameters as described in Section IV-A. The interceptor employs the optimal waveform parameters $\gamma_{k}^{*}$ to design and transmit a waveform to obtain the measurement $\mathbf{y}_{k}$. Then the state estimate update $\hat{\mathbf{e}}_{k \mid k}$ is computed as in the $\mathrm{KF}$, from the approximating likelihood $\mathcal{N}\left(\mathbf{y}_{k} ; \mathbf{e}_{k}, \tilde{\mathbf{\Phi}}_{k}\right)$ and the predictive posterior $\mathcal{N}\left(\mathbf{e}_{k} ; \mathbf{e}_{k \mid k-1}, \mathbf{P}_{k \mid k-1}\right){ }^{4}$

At each time step, the approximated posterior is Gaussian and, therefore, we can solve the control problem using the LQG solution (that is, by applying the KF on the linearised model). The optimal guidance $\mathbf{u}_{k}^{*}$ can then be computed analytically as described in Section IV-A.

\section{A. Approximated optimal waveform and guidance}

An approximation of the solution of the waveform and guidance control problem (10) can be computed applying the optimal solutions [14, Th.1] on the statistical linearised model.

Proposition 1. Under the assumption that the noises $\boldsymbol{\xi}_{k}\left(\gamma_{k}\right)$, $\mathbf{w}_{k}$ and the initial state $\mathbf{e}_{0}$ are jointly independent for $k=$ $0,1,2, \ldots$, the optimal solution of the problem (10) assuming the dynamics (1) and the linearised measurement equation (11) is $\mathbf{u}_{i}^{*}=-\mathbf{L}_{i} \hat{\mathbf{e}}_{i}$ and $\boldsymbol{\gamma}_{i}^{*}=\arg \min _{\boldsymbol{\gamma}_{i}} \operatorname{Tr}\left(\mathbf{M}_{i} \mathbf{P}_{i \mid i}\right)+\alpha_{i} \Psi_{s}\left(\boldsymbol{\gamma}_{i}\right)$ with

$$
\mathbf{M}_{i}= \begin{cases}\mathbf{S}_{N}, & \text { for } i=N \\ \left(\mathbf{E}_{i}+\mathbf{F}_{i}^{T} \mathbf{S}_{i+1} \mathbf{F}_{i}-\mathbf{S}_{i}\right), & \text { for } i=1, \ldots, N-1\end{cases}
$$

and

$$
\hat{\mathbf{e}}_{i+1}=\mathbf{F}_{i} \hat{\mathbf{e}}_{i}+\mathbf{B}_{i} \mathbf{u}_{i}+\mathbf{K}_{i+1}\left(\mathbf{y}_{i+1}-\mathbf{H}_{i+1}\left(\mathbf{F}_{i} \hat{e}_{i}+\mathbf{B}_{i} \mathbf{u}_{i}\right)\right)
$$

with $\hat{\mathbf{e}}_{0}=\mathcal{E}\left[\mathbf{e}_{0}\right]$. The Kalman gain equals

$$
\mathbf{K}_{i}=\mathbf{P}_{i \mid i-1} \mathbf{H}_{i}^{\mathrm{T}}\left(\mathbf{H}_{i} \mathbf{P}_{i \mid i-1} \mathbf{H}_{i}^{\mathrm{T}}+\boldsymbol{\Phi}_{i}\right)^{-1}
$$

where $\mathbf{P}_{i \mid i}$ is determined by the following Riccati matrix difference equation that runs forward in time:

$$
\mathbf{P}_{i \mid i}=\mathbf{P}_{i \mid i-1}-\mathbf{K}_{i} \mathbf{H}_{i} \mathbf{P}_{i \mid i-1}
$$

where

$$
\mathbf{P}_{i \mid i-1}=\mathbf{F}_{i-1} \mathbf{P}_{i-1 \mid i-1} \mathbf{F}_{i-1}^{\mathrm{T}}+\mathbf{Q}_{i-1}
$$

\footnotetext{
${ }^{4}$ To increase the accuracy of the posterior approximation, we can perform iterated statistical linearisation [19], where we set the prior moments $\mathbf{e}_{o}=$ $\mathbf{e}_{k \mid k}, \mathbf{P}_{o}=\mathbf{P}_{k \mid k}$ to recompute the approximating covariance $\boldsymbol{\Phi}_{k}$. This approach is called iterated posterior linearisation in [19]. In Section V we show that the standard statistical linearisation already provides a very good approximation for our problem and so we do not perform iterated posterior linearisation.
}

and $\mathbf{P}_{0 \mid 0}=\mathcal{E}\left(\left(\mathbf{e}_{0}-\hat{\mathbf{e}}_{0}\right)\left(\mathbf{e}_{0}-\hat{\mathbf{e}}_{0}\right)^{\mathrm{T}}\right)$. The feedback gain matrix equals

$$
\mathbf{L}_{i}=\left(\mathbf{B}_{i}^{\mathrm{T}} \mathbf{S}_{i+1} \mathbf{B}_{i}+\mathbf{R}_{i}\right)^{-1} \mathbf{B}_{i}^{\mathrm{T}} \mathbf{S}_{i+1} \mathbf{F}_{i}
$$

where $\mathbf{S}_{i}$ is determined by the following Riccati matrix difference equation that runs backward in time:

$\mathbf{S}_{i}=\mathbf{F}_{i}^{\mathrm{T}}\left(\mathbf{S}_{i+1}-\mathbf{S}_{i+1} \mathbf{B}_{i}\left(\mathbf{B}_{i}^{\mathrm{T}} \mathbf{S}_{i+1} \mathbf{B}_{i}+\mathbf{R}_{i}\right)^{-1} \mathbf{B}_{i}^{\mathrm{T}} \mathbf{S}_{i+1}\right) \mathbf{F}_{i}+\mathbf{E}_{i}$

with $\mathbf{S}_{N}=\mathbf{E}_{N}$. Finally the optimal cost is

$$
\begin{aligned}
& \min _{\mathbf{u}_{0: N-1}, \boldsymbol{\gamma}_{1: N}} J\left(\mathbf{e}_{0}, \mathbf{u}_{0: N-1}, \boldsymbol{\gamma}_{1: N}\right)=\mathcal{E}\left(\mathbf{e}_{0}^{T} \mathbf{S}_{0} \mathbf{e}_{0}\right)+\operatorname{Tr}\left(\mathbf{M}_{N} \mathbf{P}_{N \mid N}^{*}\right) \\
& +\alpha_{N} \Psi_{s}\left(\boldsymbol{\gamma}_{N}^{*}\right)+\sum_{i=0}^{N-1} \operatorname{Tr}\left(\mathbf{S}_{i+1} \mathbf{Q}_{i}\right)+\operatorname{Tr}\left(\mathbf{M}_{i} \mathbf{P}_{i \mid i}^{*}\right)+\alpha_{i} \Psi_{s}\left(\boldsymbol{\gamma}_{i}^{*}\right)
\end{aligned}
$$

where $\mathbf{P}_{i \mid i}^{*}$ is the value of the covariance matrix $\mathbf{P}_{i \mid i}$ when computed with the optimal measurement noise covariance $\boldsymbol{\Phi}_{i}^{*}$, that is computed using the optimal waveform parameters $\left(\gamma_{i}^{*}\right)$.

Due to statistical linearisation, the solution is only an approximation of the optimal strategy. Nonetheless, we will show in the next section that, in practice, it is very close to the optimal one.

\section{Simulation Results}

We consider a moving airborne platform that carries a pulsed Doppler radar system operating at $f_{0}=10 \mathrm{GHz}$ with the task of reaching a target within 50 radar scans. The dynamical and measurement equations are reported in Section II.

At each scan the radar transmits $N_{P}=2$ pulses with a PRI of $0.1 \mathrm{~ms}(\mathrm{PRF}=10 \mathrm{kHz})$. The transmitting and receiving antenna gain is $G=10 \mathrm{~dB}$ and the target RCS is $1 \mathrm{~m}^{2}$. The SNR is calculated at each $k$-th scan, using the real target relative position $r(k)$ and the selected waveform parameter $\lambda_{G}(k)$ in the radar SNR equation in (8), assuming a receiver equivalent noise temperature $T_{e}=1000 \mathrm{~K}^{\circ}$ and a peak power $P_{p k}=100 \mathrm{~W}$. As a result, whilst the term $\psi_{s}(k)$ is a function of the waveform parameters alone, the covariance of the measurement noise becomes a function of the state. We also assume the transmitter and receiver employ separate antennas that point at the target with $\sigma_{\theta}^{2}=0.1 \mathrm{rad}^{2}$ so that the radar can transmit and receive at all times with no blind range. The initial state $\mathbf{e}_{0}$ is $5 \mathrm{~km},-1 \mathrm{~m} / \mathrm{s}, 0 \mathrm{rad}$ and $0 \mathrm{rad} / \mathrm{s}$. The covariance matrix for the $\mathrm{KF}$ is set to $\mathbf{P}_{0 \mid 0}=\operatorname{diag}(100,10,1,1)$ and the initial state estimate is sampled from a multivariate Gaussian distribution with mean $\mathbf{e}_{0}$ and covariance $\mathbf{P}_{0 \mid 0}$. Finally, the weight $\alpha_{k}=2 \times 10^{3}$, the weight matrices are $\mathbf{E}=\mathbf{I}_{4 \times 4}$, $\mathbf{S}_{N}=8 \times 10^{3} \mathbf{I}_{4 \times 4}$ and $\mathbf{R}=8 \times 10^{3} \mathbf{I}_{4 \times 4}$.

Results of the proposed approach, JWGCO/SL, are compared with three other strategies:

MinN: $\quad \min _{\boldsymbol{\gamma}_{k}} \operatorname{Tr}\left(\boldsymbol{\Phi}_{k}\right)$
MinP: $\min _{\boldsymbol{\gamma}_{k i}} \operatorname{Tr}\left(\mathbf{E} \mathbf{P}_{i \mid i}\right)$
JWGCO oracle 
In the first strategy, the waveform parameters are selected so to minimize the trace of the expected measurement noise covariance matrix $\mathcal{E}\left(\mathbf{N}\left(\mathbf{e}_{k}, \gamma\right)\right)$, while, in the second strategy, we minimise at each scan the trace of $\mathbf{P}_{i \mid i}$ weighted by the matrix E. The third strategy is JWGCO computed using the true unknown covariance matrix $\mathbf{N}\left(\mathbf{e}_{k}, \gamma\right)$ (that is we used the true range to compute the covariance matrix $\mathbf{N}\left(\mathbf{e}_{k}, \gamma_{k}\right)$ We call this last strategy "JWGCO-oracle" and it is unattainable in practice..$^{5}$

The bandwidth and pulsewidth of the chirp pulse at each scan are automatically selected by the algorithm in the range between $10 \mathrm{MHz}$ to $50 \mathrm{MHz}$ and $1 \mu$ s to $10 \mu$ s, respectively. The estimated relative velocity versus the relative range to the target for the four waveform selection strategies is showed in Figure 1. Results have been averaged over 500 Monte Carlo simulations. The interceptor reaches the target within

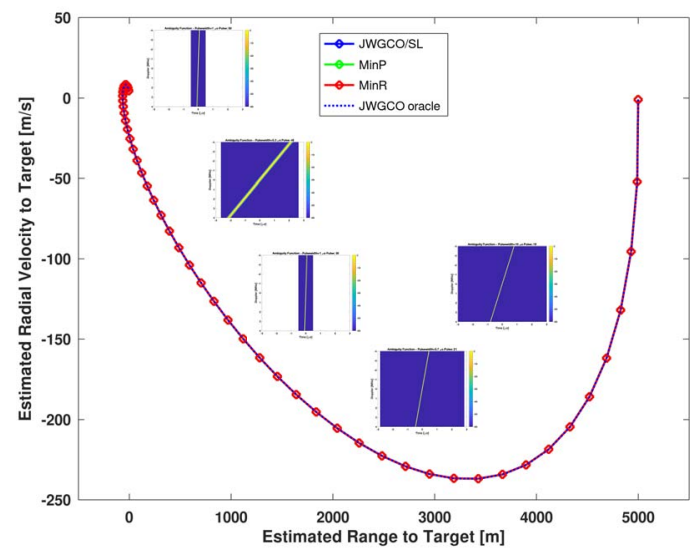

Fig. 1. Estimated relative velocity versus range to target for the four waveform selection strategies.

the predefined number of scans for all cases. Figure 2 shows the optimal pulsewidth selected by the four strategies at each scan. Results show that the JWGCO/SL algorithm selects the short pulsewidth after scan \#21. Note that there is practically no difference between JWGCO/SL and JWGCO-oracle, which shows that the statistical linearisation approach proposed in this paper (to deal with the dependence on the state of the measurement noise) allows the interceptor to select the optimal pulsewidth at each scan. The bandwidth is fixed at all scans indicating no variation in range resolution (we did not report the figure). As a result of the selected waveform parameters, the ambiguity function rotates during the trajectory as shown in Fig. 1.

Figure 3 shows the averaged cumulative cost, i.e. the incremental value of (10) as $k \rightarrow N=50$ for the last 20 scans. As expected, the strategies JWGCO achieves the lowest final minimum cumulative cost with respect to MinR and MinP strategies. The contribution to the total cost of the transmitted energy, estimation errors and control input terms are shown

\footnotetext{
${ }^{5}$ It is the best unattainable statistical linearisation.
}

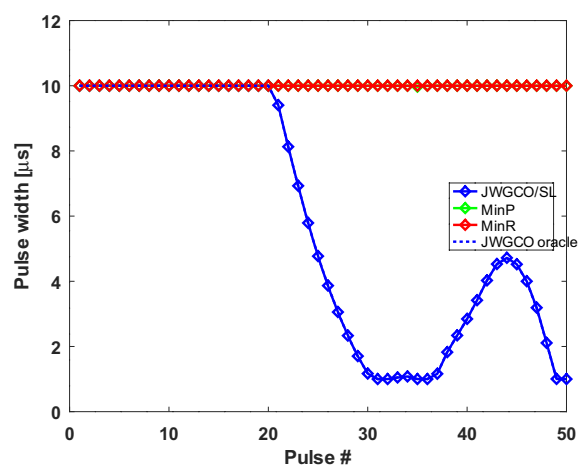

Fig. 2. Pulsewidth of the resulting linear chirp for the three waveform selection strategies at each scan.

in Figure 4. The left plot shows that the JWGCO results in a series of waveforms with the lowest transmitted energy.

Figures 5 show the Root Mean Square Error (RMSE) of the estimate of relative range, relative radial velocity and relative angle, respectively.

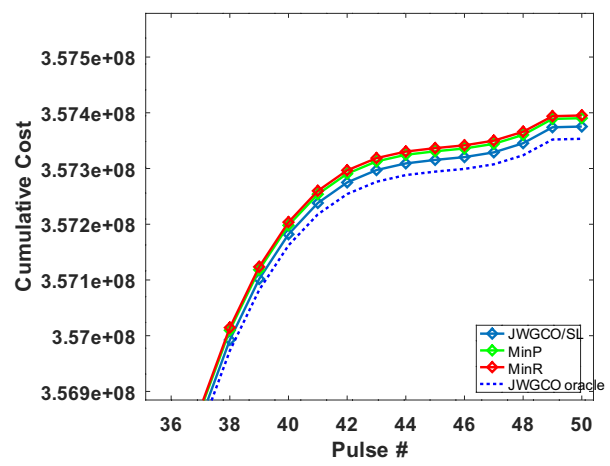

Fig. 3. Average cumulative cost for the three strategies.

\section{CONCLUSIONS}

A solution based on Statistical Linearisation (SL) has been presented that extends the JWGCO algorithm to the non-linear case. The proposed algorithm accounts for the dependency of the radar measurement accuracies on the target position and, as a direct consequence, on the Signal to Noise Ratio (SNR). Performance is assessed with a set of simulations for a pulsed Doppler radar transmitting linearly frequency modulated chirps.

\section{REFERENCES}

[1] A. Farina, A. De Maio, and S. Haykin, Eds., The Impact of Cognition on Radar Technology, ser. Radar, Sonar and Navigation. Institution of Engineering and Technology, 2017.

[2] A. Charlish, K. Bell, N. Godman, and G. Smith, "Special issue on cognitive radar," IET Radar, Sonar and Navigation, vol. 12, no. 12, pp. 1361-1475, 2018.

[3] D. Kershaw and R. Evans, "Optimal waveform selection for tracking systems," IEEE Transactions on Information Theory, vol. 40, no. 5, pp. 1536-1550, Sep 1994. 

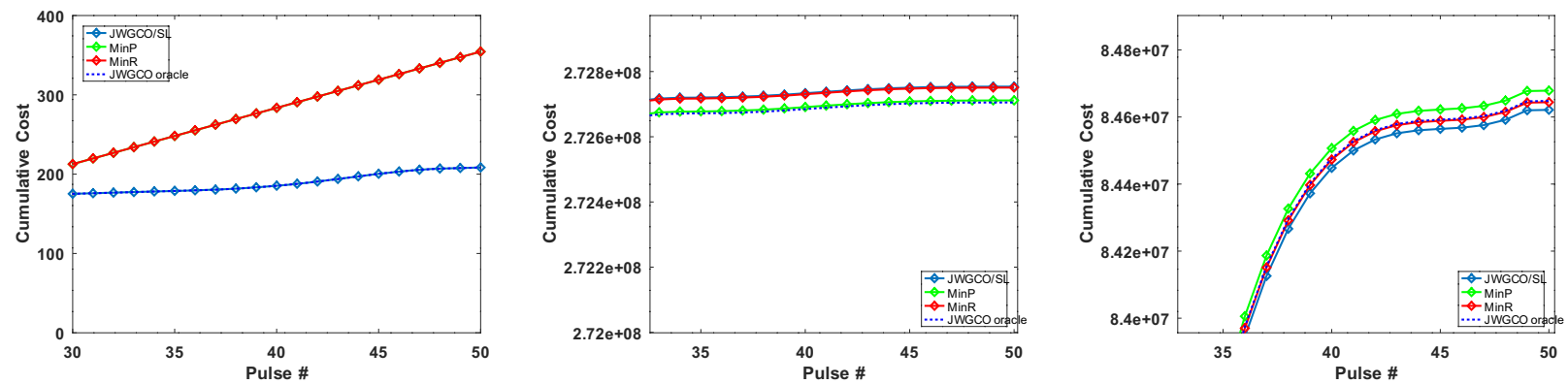

Fig. 4. Average cumulative cost of the term associated with the transmitted energy (left), estimation errors (center) and control input term (right).
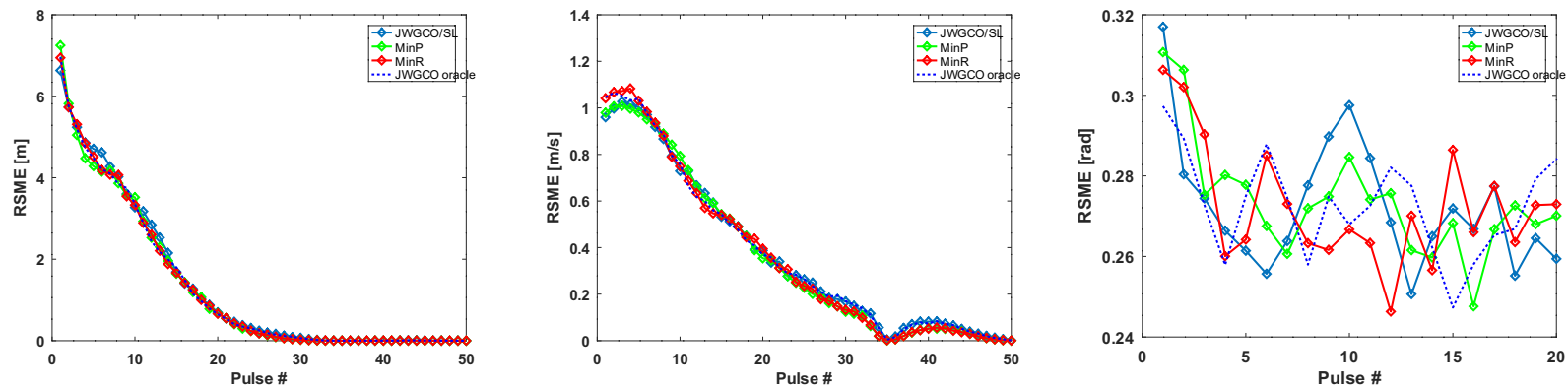

Fig. 5. Root Mean square Error of the estimate of relative range, relative radial velocity and relative angle

[4] R. Niu, P. Willett, and Y. Bar-Shalom, "Tracking considerations in selection of radar waveform for range and range-rate measurements," IEEE Transactions on Aerospace and Electronic Systems, vol. 38, no. 2, pp. 467-487, Apr 2002.

[5] S. P. Sira, A. Papandreou-Suppappola, and D. Morrell, "Dynamic configuration of time-varying waveforms for agile sensing and tracking in clutter," IEEE Transactions on Signal Processing, vol. 55, no. 7, pp. 3207-3217, July 2007.

[6] M. Hurtado, T. Zhao, and A. Nehorai, "Adaptive polarized waveform design for target tracking based on sequential Bayesian inference," IEEE Transactions on Signal Processing, vol. 56, no. 3, pp. 1120-1133, March 2008.

[7] K. L. Bell, C. J. Baker, G. E. Smith, J. T. Johnson, and M. Rangaswamy, "Cognitive radar framework for target detection and tracking," IEEE Journal of Selected Topics in Signal Processing, vol. 9, no. 8, pp. 14271439, Dec 2015.

[8] A. E. Mitchell, G. Smith, K. L. Bell, and M. Rangaswamy, "Cost function design for the fully adaptive radar framework," IET Radar, Sonar and Navigation, vol. 12, no. 12, pp. 1380-1389, 2018.

[9] S. Sen and A. Nehorai, "OFDM MIMO radar with mutual-information waveform design for low-grazing angle tracking," IEEE Transactions on Signal Processing, vol. 58, no. 6, pp. 3152-3162, June 2010.

[10] S. Wang, D. Bi, H. Ruan, and S. Chen, "Cognitive structure adaptive particle filter for radar manoeuvring target tracking," IET Radar, Sonar and Navigation, vol. 13, no. 1, pp. 23-30, 2019.

[11] N. H. Nguyen, K. Doğançay, and L. M. Davis, "Joint transmitter waveform and receiver path optimization for target tracking by multistatic radar system," in 2014 IEEE Workshop on Statistical Signal Processing (SSP), June 2014, pp. 444-447.

[12] M. Athans, "On optimal allocation and guidance laws for linear interception and rendezvous problems," IEEE Transactions on Aerospace and Electronic Systems, vol. AES-7, no. 5, pp. 843-853, Sept 1971.

[13] A. Balleri, A. Farina, and A. Benavoli, "Coordination of optimal guidance law and adaptive radiated waveform for interception and rendezvous problems," IET Radar, Sonar and Navigation, vol. 11, no. 7, pp. 1132-1139, 2017.

[14] A. Benavoli, A. Balleri, and A. Farina, "Joint waveform and guidance control optimization for target rendezvous," IEEE Transactions on Signal Processing, vol. 67, no. 16, pp. 4357-4369, 2019.
[15] L. Ortenzi, L. Timmoneri, and D. Vigilante, "Unscented Kalman Filter (UKF) applied to FM band passive radar," in 2009 International Radar Conference "Surveillance for a Safer World" (RADAR 2009), Bordeaux, France, Oct 2009, pp. 1-6.

[16] B. Ristic, S. Arulampalam, and N. Gordon, Beyond the Kalman Filter: Particle Filters for Tracking Applications. Artech House, 2004.

[17] A. Balleri and A. Farina, "Ambiguity function and accuracy of the hyperbolic chirp: comparison with the linear chirp," IET Radar, Sonar and Navigation, vol. 11, no. 1, pp. 142-153, 2017.

[18] K. J. Åström, Introduction to stochastic control theory. Courier Corporation, 2012.

[19] Á. F. García-Fernández, L. Svensson, M. R. Morelande, and S. Särkkä, "Posterior linearization filter: Principles and implementation using sigma points," IEEE Transactions on Signal Processing, vol. 63, no. 20, pp. 5561-5573, 2015.

[20] S. J. Julier and J. K. Uhlmann, "Unscented filtering and nonlinear estimation," Proceedings of the IEEE, vol. 92, no. 3, pp. 401-422, 2004 